\title{
24
}

\section{Current Understanding and Quantification of Clouds in the Changing Climate System and Strategies for Reducing Critical Uncertainties}

\author{
Johannes Quaas, Rapporteur \\ Sandrine Bony, William D. Collins, Leo Donner, \\ Anthony Illingworth, Andy Jones, Ulrike Lohmann, \\ Masaki Satoh, Stephen E. Schwartz, \\ Wei-Kuo Tao, and Robert Wood
}

\begin{abstract}
To date, no observation-based proxy for climate change has been successful in quantifying the feedbacks between clouds and climate. The most promising, yet demanding, avenue to gain confidence in cloud-climate feedback estimates is to utilize observations and large-eddy simulations (LES) or cloud-resolving modeling (CRM) to improve cloud process parameterizations in large-scale models. Sustained and improved satellite observations are essential to evaluate large-scale models. A reanalysis of numerical prediction models with assimilation of cloud, aerosol, and precipitation observations would provide a valuable dataset for examining cloud interactions. The link between climate modeling and numerical weather prediction (NWP) may be exploited by evaluating how accurate cloud characteristics are represented by the parameterization schemes in NWP models.

A systematic simplification of large-scale models is an important avenue to isolate key processes linked to cloud-climate feedbacks and would guide the formulation of testable hypotheses for field studies.

Analyses of observation-derived correlations between cloud and aerosol properties in combination with modeling studies may allow aerosol-cloud interactions to be
\end{abstract}


detected and quantified. Reliable representations of cloud dynamic and physical processes in large-scale models are a prerequisite to assess aerosol indirect effects on a large scale with confidence.

To include aerosol indirect effects in a consistent manner, we recommend that a "radiative flux perturbation" approach be considered as a complement to radiative forcing.

\section{Are There Observational Proxies in the Present- day Climate for Future Cloud Perturbations?}

Climate sensitivity, defined as the equilibrium change in global mean temperature in response to a doubling in the atmospheric $\mathrm{CO}_{2}$ concentration, is still a very uncertain quantity (e.g., Randall et al. 2007). The primary reason for the spread in climate sensitivities, as simulated by different global climate models, is the difference in the representation of cloud processes and cloud-climate feedbacks (Soden and Held 2006; Dufresne and Bony 2008). Cloud processes determine the amount and distribution of precipitation, which is a key parameter for land-atmosphere interactions, for carbon-climate feedbacks, and for climate impact studies (e.g., water resources). Moreover, the representation of cloud microphysical properties is critical for the simulation of interactions between clouds and aerosols. Improving how cloud processes are represented in global climate models is thus of paramount importance, not only for estimates of climate sensitivity but also for projections of future climate change and their use in impact studies.

\section{Limitations of Observational Proxies}

Since cloud and radiation observations are available on a global scale for only a climatically short time period (at best, ca. 25 years), our ability to observe cloud-climate feedbacks directly is limited. Thus the question arises as to whether there might be some observational proxies in the present-day climate for future cloud perturbations. The dynamic and thermodynamic forcings of clouds that occur on shorter timescales do not correspond directly to the dynamic and thermodynamic changes that are expected to occur with global warming. Therefore, neither the seasonal cycle, which is useful to constrain the snow/ice albedo feedback (Hall and Qu 2006), nor El Niño/La Niña events can be considered as good analogues of long-term climate changes for the analysis of cloud-climate feedbacks. This was confirmed by the FANGIO study (Cess et al. 1990), which found no direct relationship between cloud seasonal variations and the cloud response to climate change. Based on a study comparing the response of clouds simulated by a climate model in the case of a volcanic eruption (Mt. Pinatubo) or of a doubling $\mathrm{CO}_{2}$ experiment (Yokohata et al. 2005), it appears that volcanic eruptions may also not be considered as useful proxies for future cloud changes, despite the success of studies constraining 
the water vapor feedback (Soden et al. 2002). Thus, no direct observational proxy for future cloud changes has yet been identified.

A better understanding of the physical processes that control cloud-climate feedbacks may, however, help uncover new pathways to exploit existing observational datasets for the understanding of cloud-climate feedbacks.

\section{Options for the Exploitation of Observational Data}

Compositing techniques can be used to compare models and observations in a way that can be relevant for evaluating cloud-climate feedbacks (see Illingworth and Bony, this volume). These methodologies permit an assessment of how clouds change with dynamic and thermodynamic conditions in the present-day climate (e.g., Bony et al. 2004). In these studies, it is essential to eliminate, as much as possible, the dynamic contribution to observed relationships between cloud properties and temperature. Other studies have decomposed the global cloudiness into a small number of prominent cloud regimes and used this decomposition to understand and assess the response of clouds to long-term climate changes (e.g., Williams and Tselioudis 2007). Of course, a perturbed climate will be characterized by both perturbed thermodynamics and dynamics, both of which are a challenge to model.

Rapid changes in clouds as a response to instantaneous $\mathrm{CO}_{2}$ doubling have been identified by Gregory and Webb (2008) in a slab model ensemble. Changes in radiative cooling in the atmosphere and fast surface responses, which are associated with the $\mathrm{CO}_{2}$ change, operate on timescales of months; that is, before variations in longer-term sea surface temperature (SST) are established. Such changes constitute a substantial and, in some cases, dominant fraction of longer-term changes. They may be detectable in the satellite record of changes of cloud forcing. However, issues related to confounding changes in the atmosphere (e.g., attributable to aerosols) may complicate detection of a signal.

The fluctuation dissipation (FD) theorem states that the transient behavior (and sensitivity) of dynamic systems to perturbations can be determined from their natural variability. This theorem has been adapted from statistical physics, where it applies to a wide array of classical and quantum mechanical systems. It provides a potential mechanism for estimating or constraining climate sensitivity from short-term unforced variations in the climate system (Leith 1975; Schwartz 2007). To date, however, there is no rigorous determination of whether the FD theorem is applicable to the Earth's climate and, if so, which fields and fluctuations constrain climate sensitivity. The prospect that the theorem might apply to spectral features of the Earth's radiation field is under active investigation. 


\section{Process-based Evaluation of Large-scale Models to Gain Confidence in Cloud Feedback Determination}

Cloud-climate feedbacks can be simulated with general circulation models (GCMs). To gain confidence in the results from these simulations, the reliability of the physics of climate models (e.g., the representation of turbulence, convection, aerosols, and clouds) must be improved (Illingworth and Bony, this volume). For this purpose, a well-recognized methodology, which forms the basis of the GEWEX cloud system studies, can be employed: the physics of climate models within a single-column framework are compared with observations from field experiments and/or LES or CRM simulations driven by observed forcings (Browning et al. 1993; Randall et al. 2003). The resulting parameterizations are then evaluated in a full 3-D GCM with global datasets to assess whether an improvement of cloud representation has been achieved. Such an approach can be very powerful in pointing out deficiencies in model parameterizations and in improving model parameterizations.

Satellite observations provide particularly well-suited datasets to evaluate GCM processes, because of their global coverage and the availability of large statistics (Illingworth and Bony, this volume). Recent spaceborne active remote-sensing data providing information about the vertical distribution of cloud-related quantities (CALIPSO, CloudSat), as well as precipitation radar (TRMM) and advanced passive instrument retrievals, are especially valuable to enhance the understanding of processes and enable the evaluation of climate models. This, in turn, has enhanced our understanding of processes and enabled the evaluation of climate models. The development and application of satellite simulators in climate models that predict sensor responses assures the comparability of simulations with actual observations (Webb et al. 2001; Bodas-Salcedo et al. 2008; Chepfer et al. 2008). A persistent shortcoming, however, is the lack of high-resolution water vapor retrievals and its probability density function (PDF), which forms the basis of statistical cloud parameterization schemes and has a large impact on cloud processes. Potentially, differential absorption and Raman lidar technology should help improve this situation in the future.

The general consistency of climate models with the global atmospheric models used for NWP suggests that it is possible to evaluate climate models in the NWP framework. Comparison of the representation of clouds in NWP models (or GCMs run in NWP mode) with observations (e.g., satellite data or networks of instrumental sites such as Cloudnet; Illingworth et al. 2007) can identify strengths and shortcomings in NWP parameterization schemes. Parameter values that are applied in parameterization schemes used in an NWP model run with data assimilation may be rejected if they lead to systematic and unrealistic tendencies in short-term forecasts (Rodwell and Palmer 2007). This technique might be adapted to identify features or errors in short-term cloud simulations that map directly onto features in long-term cloud radiative feedbacks. 
Systems to predict the climate evolution on a decadal timescale are currently under development. Once in place, they may permit hindcast simulations to be performed, from which information about cloud-climate feedbacks could be inferred.

Despite the fundamental importance of physical parameterizations to improve climate model simulations and reduce uncertainties in climate projections, very few people are actively involved in the development, evaluation, and improvement of physical parameterizations. Progress in developing reliable model-based projections of future climate change could be greatly enhanced by these activities, for which considerable time and energy are required.

\section{Essential Observations}

The Oklahoma Atmospheric Radiation Measurement (ARM) site, which has been operating for 15 years, provides an example of detailed ground-based cloud process observations over long time series. Although these measurements contribute greatly to the improvement of process understanding and the development of process representations in models, studies that provide reliable information about cloud-climate feedbacks derived directly from these data are still lacking. One reason might be that the available data relate only to a specific, individual location. An ensemble of dedicated ground-based measurement sites positioned over a large spatial scale and over a long time period could resolve this issue. Such sites might be less well equipped than ARM or Cloudnet sites. However, as a minimum requirement, they would need to observe some main cloud properties (e.g., cloud-base and cloud-top heights, integrated liquid water path (LWP), radiative cooling and fluxes, precipitation) together with thermodynamic properties derived from radiosondes. Maintaining such an ensemble for a few years (to encompass, e.g., El Niño and La Niña events) could provide the opportunity to explore relationships between cloud properties, large-scale atmospheric circulation, and thermodynamic stratification of the atmosphere (e.g., low-level tropospheric static stability, low-level humidity gradients).

For cloud-climate feedback studies, a sustained long-term monitoring of the Earth's radiation budget (ERB) is essential. Loeb et al. (2007) suggest that with the current measurement stability of broadband radiometers, such as CERES, a record of about ten years of consistent observations would allow for the detection of some cloud-climate feedbacks. Since a substantial part of cloud feedbacks act on longer timescales, sustained ERB observation is necessary. The commitments to operate broadband radiometers onboard the EarthCARE and NPOESS NPP platforms are important, as are the contributions of instruments monitoring ERB at high temporal resolution. However, for the latter, spatial coverage is limited (e.g., GERB on MSG and ScaRaB on Mega-Tropique). In addition, data are not able to be intercompared as a result of possible instrument failures, suggesting the need for an absolutely calibrated 
ERB instrument. Retrievals with such an instrument at any two times could then be compared to estimate the temporal change in the ERB.

A dataset of reanalyses, including the assimilation of satellite observations of cloudy atmospheres, may prove particularly useful for the investigation of cloud-climate feedbacks.

\section{Summary}

Cloud-climate feedbacks represent a major uncertainty in projections of climate change. Proxies for climate change (e.g., seasonal cycle, interannual variability, volcanic eruptions) have thus far been found to be unfit to quantify cloud feedbacks. Improved understanding of the cloud-climate feedback processes may lead to a better exploitation of existing data. Compositing techniques to separate feedback mechanisms, as well as the combination of model results with observational data, have already enhanced our understanding of cloud feedbacks. New analysis methods of cloud responses to forcing in models, and of natural variability, may yield further insights. A very promising, though demanding pathway is to improve cloud process parameterizations in large-scale models through the use of observations and LES or CRM, as well as by exploiting NWP experiences. Sustained observations from existing and additional satellites and ground-based sites are needed to support these approaches.

\section{What Are the Limitations of Bottom-up Diagnoses that Require Top-down Approaches?}

\section{Limitations of Current Small- to Mesoscale Observations}

Traditional field programs have advanced our process-level understanding of the climate system and the representation of cloud processes in detailed models. Still, it has proven far more challenging to apply the field observations to constrain the parameterizations and large-scale properties of clouds in models. Challenges arise from the disparities in spatial scales between point measurements and climate models (the so-called "process-parameterization gap") as well as from disparities in temporal scales between short-term field programs and long-term climate change.

To engage climate model development with field measurements, we suggest that sensitivity studies with large-scale models be used more frequently to guide the design of field programs. For example, intermodel differences across ensembles of climate models could be used to identify processes, cloud systems, or aerosol-cloud interaction processes that represent major sources of uncertainty in simulations of climate perturbations. These differences could then be targeted for detailed field programs. Consideration should be given to 
methods that bridge the spatial and temporal gaps, so that field measurements could be used to test climate sensitivity.

\section{Simplification of Large-scale Models to Guide Process Understanding}

Over the last decade, the climate modeling community has increased the complexity of models to address critical scientific questions by adding more processes or components (e.g., aerosols, chemistry, carbon cycle, dynamic vegetation) and increasing the model resolution. It appears, however, that this did not yield a better understanding of cloud-climate feedbacks, because the diagnosed uncertainty in climate sensitivity has not decreased with time. Large-scale models appear most useful not only as a basis for the quantification of climate sensitivity but also as a framework for advancing ideas and understanding on how the climate works and responds to external perturbations. Such models can be used to develop concepts, which in turn must then be explored, or isolated, through simplifications.

A better understanding of the physical mechanisms underlying the cloudclimate feedbacks produced by climate models could be useful in designing a strategy to evaluate these feedbacks using observations. By simplifying (rather than complicating) models and conducting idealized experiments, we may be able to pinpoint the main critical processes, to prioritize them, and to test resulting ideas or theories. Interpretation frameworks could then be proposed to assist our understanding of the physics of and intermodel differences in cloudclimate feedbacks.

One approach to simplify GCMs may be to reduce the complexity of the large-scale boundary conditions (e.g., aquaplanet versions of the models, even for CRMs or super-parameterizations), to reduce the dimensionality of the system (e.g., 2-D or 1-D model versions derived from the 3-D model), or even to remove some processes (i.e., replace complex microphysical schemes with simpler ones). Models of intermediate complexity, such as the quasi-equilibrium tropical circulation model (Neelin and Zeng 2000), might also be used. Simple conceptual models (e.g., 2-box models) may be viewed as the ultimate step to this simplification process.

The extent to which simplified models are useful in reproducing and interpreting complex model results should be tested and quantified by analyzing, for a given model, how the cloud-climate feedbacks compare across the hierarchy of model complexities. An ideal situation would be for each GCM to be associated with a suite of simpler or more idealized model versions to support the analysis and the understanding of its results. 


\section{Ensembles of Process Model Simulations to Assess Large-scale Cloud Feedbacks}

Systematic comparisons between process models (LES and CRM) and largescale models may be used to analyze and improve cloud parameterizations in an effort to assess cloud-climate feedbacks on a large scale (discussed above). Idealized sensitivity experiments (e.g., +2 K SST perturbations or perturbations applied to cloud properties) might be conducted using LES or CRMs (i.e., in the absence of any interaction with the large-scale circulation) and compared to GCM simulations allowing for this interaction.

Comparing the response of clouds at these different scales would enable us to understand the response of clouds to a perturbation at a specific scale as well as the various controlling factors involved. Global CRMs or superparameterization models constitute integrating tools of small and large spatial scales and may play a particularly important role in this regard (Collins and Satoh, this volume).

\section{Summary}

For observational studies to be successful, key mechanisms of cloud-climate feedbacks need to be identified. This can be accomplished by simplifying largescale models. Systematic comparisons between process and large-scale models offer the best opportunity to improve the representation of cloud processes in models and to analyze cloud feedback processes.

\section{Is the Uncertainty Range of Radiative Flux Perturbations by Aerosols Related to Systematic Errors in Climate State? What Aspects of Clouds Must Be Represented to Treat Cloud-Aerosol Interactions with Fidelity?}

\section{Uncertainties in Estimates of Radiative Flux Perturbations}

Aerosol forcing, as inferred from representing the geographical distribution of anthropogenic aerosols and their radiative influences in climate models (i.e., the so-called forward estimates of forcing), has been consistently larger than "inverse" estimates, whereby aerosol forcing is inferred from the total forcing required to obtain the observed temperature change over the industrial period for the generally accepted range of climate sensitivity, observed ocean heat uptake, and given anthropogenic greenhouse gas forcing (Anderson et al. 2003). Given the large uncertainties in processes connected to both aerosol forcings and climate sensitivity, it is not obvious that this discrepancy can simply be attributed to an overestimate of the aerosol forcings by forward estimates. 
In a multimodel comparison, Denman et al. (2007) analyzed the forcing of anthropogenic aerosols with respect to the aerosol compounds and aerosolcloud interaction processes considered (i.e., whether or not aerosol interactions with mixed-phase and ice clouds are taken into account). If sulfate or sulfate and black carbon are solely used, they found that global mean forcing is larger than if organics and aerosol interactions with mixed-phase and ice clouds are included as well. However, the variations within a given aerosol compound category are at least as large as the differences between different categories.

Calculations of the total aerosol forcing depend critically on estimates of both present-day and preindustrial aerosol (precursor) emissions. In particular, the limited knowledge of preindustrial aerosol concentrations introduces considerable uncertainty about the radiative forcing by aerosols. This is manifest in our lack of knowledge about the physical processes that determine the unperturbed cloud droplet number concentration (CDNC) for use in global climate model parameterizations. Observations show that $\mathrm{CDNC}$, even in very clean regions, falls rarely below some lower bound (approximately $10 \mathrm{~cm}^{-3}$ ).

\section{Cloud Aspects Critical for Aerosol Indirect Effect Quantifications}

\section{Cloud Albedo}

The sensitivity of cloud albedo to perturbations in cloud condensation nuclei, and thus the strength of the Twomey effect (Twomey 1974), depends on the value of cloud albedo. To simulate aerosol indirect effects with fidelity, we must thus be able to simulate realistically the distribution of cloud albedo (Feingold and Siebert, this volume). Satellite data of this quantity exist, allowing for an observation-based evaluation of the distribution.

\section{Boundary Layer Moisture Budget}

Process modeling and theoretical studies suggest that for stratocumulus, the cloud thickness response to perturbations in CDNC may, in many cases, act to oppose the changes in albedo caused by the Twomey effect alone. The dynamic and microphysical processes responsible for this depend critically on surface relative humidity. In general, the total aerosol indirect effect may be strongly sensitive to the cloud macrophysical and boundary layer properties (see Brenguier and Wood; Feingold and Siebert; and Stevens and Brenguier, all this volume).

\section{Cloud Subgrid-scale Variability}

The aerosol and microphysical processes that determine cloud radiative properties occur on scales much smaller than those resolved by GCMs, and they 
are nonlinear. Thus, the application of GCM-resolved moisture and motion fields will produce unrealistic estimates of aerosol indirect effects. A general approach to address this problem is to construct PDFs for subgrid velocity and moisture distributions (Lohmann and Schwartz, this volume). Aerosol and microphysical processes are then evaluated using the PDF vertical velocity and moisture.

\section{Microphysical Processes in Ice and Mixed-phase Clouds}

Aerosols may have an important influence on the optical properties of ice clouds as well as on microphysical processes in mixed-phase clouds. To simulate such effects realistically, the various ice crystal nucleation pathways and mixed-phase microphysical processes must be accurately represented in the model (Lohmann and Schwartz, this volume).

\section{Summary}

"Inverse" estimates (i.e., aerosol forcings inferred from the observed global warming) are hampered by the uncertainty about climate sensitivity. Biases in modeled cloud fields, limited process representation, missing aerosol-cloud interaction processes, and uncertainties in the unperturbed aerosol distribution are the main sources of uncertainties for simulated aerosol forcings ("forward" estimates). Key cloud aspects necessary for a realistic representation of aerosol-cloud interactions in large-scale models are the distribution of cloud albedo, boundary layer moisture budget, subgrid-scale variability of moisture and vertical updraft velocity, as well as ice crystal nucleation and mixed-phase cloud microphysical processes. In general, the quantification of perturbations of cloud properties by anthropogenic aerosols in large-scale models is highly dependent on the formulation of the cloud process representations.

\section{Is It Possible to Design an Observational Program to Detect and Quantify Aerosol Indirect Effects?}

\section{Limits to Existing Observation-based Studies}

Despite many observational campaigns, no large body of observational evidence exists to provide a positive correlation between cloud albedo and aerosol perturbations on a large scale. Although it has been established that an increase in aerosol concentration results usually in an increase of CDNC, this does not necessarily lead to an increase in cloud albedo, since the LWP of the cloud also changes. Although the LWP may be responding to aerosol-induced 
changes, it may be determined to a first order by differences in large-scale meteorological forcings.

\section{Ship Track Analyses}

Studies of plumes that interact with clouds, as typified by ship tracks, provide a natural laboratory to sample a partial derivative with respect to aerosol concentration or cloud droplet concentration, unlike, for example, a regional-scale plume of polluted air, in which the meteorology co-varies with the aerosol properties. Heat and moisture are also injected into the atmosphere within ship exhaust, but these perturbations can be regarded as negligible after the plume spreads over a few kilometers in width. However, ship tracks occur only in one type of cloud (marine stratocumulus), and they appear to be more likely in shallow boundary layers (Coakley et al. 2000). Despite these limitations, analyses of ship tracks might be exploited to yield further insights into indirect effects of aerosols, since they reveal information about the important processes (e.g., entrainment) that control the cloud response to aerosol perturbations (see Cotton, this volume).

\section{Statistical Relationships}

Correlations between cloud properties (cloud droplet radius, CDNC) and column aerosol concentration (aerosol index or aerosol optical depth, or a proxy such as hemispheric or land-sea contrasts) from satellite data may be used to infer some clues about aerosol-cloud interactions (Nakajima and Schulz, this volume). Since it is, however, impossible with a passive satellite instrument to quantify the relevant cloud and aerosol parameters simultaneously at a particular location, these analyses rely on the assumption that aerosol properties in clear scenes are similar to those in near-by cloudy situations. Long-term measurements from ground-based sites are necessary to establish similar correlations (Feingold and Siebert, this volume).

\section{Approaches for Better Use of Observations}

\section{Analysis of Time History in Satellite Retrievals}

Observations and analyses from a combination of various satellite instruments (e.g., from the A-Train constellation) provide a comprehensive dataset for studying global cloud and aerosol properties, their radiative effects, and their interrelationship. It is critical to determine whether the correlations imply causation (e.g., whether correlations between cloud albedo and aerosol optical depth from adjacent clear-sky regions constitute evidence for aerosol indirect effects). Correct construction and interpretation of the correlations require methods for identifying measurements with similar physical and chemical 
tendencies over the lifetime of the clouds prior to measurement. These tendencies are not available from the instantaneous observations collected by polar-orbiting satellites. However, the tendencies could be constructed in a chemical and meteorological assimilation of satellite observations. Analyses of satellite data might be enhanced to include the tendencies of physical and chemical environmental factors that have been shown to govern cloud-aerosol interactions.

\section{Model-Data Comparisons to Infer Causalities}

To explore the extent to which observed correlations between cloud and aerosol properties represent causality, similar correlations can be computed in models. Consistency between a process model and observations and sensitivity studies with the model, with and without relevant processes, provide confidence that causality can be inferred from observed correlations (Feingold and Siebert, this volume).

\section{Careful Choice of Analyzed Situations}

The use of natural meteorological variability is a means of testing the ability of a model to represent correctly the key physics of the aerosol indirect effects. Careful choice needs to be made regarding possible locations where this might be beneficial. The system should have some simple and effective proxies for meteorological control, the meteorological variability must not greatly swamp the essential aerosol signal, the albedo of the clouds in the system need to be potentially susceptible to perturbations in aerosols, and the clouds in the system should have sufficient horizontal homogeneity such that their properties (microphysical and macrophysical) are detectable from space. To some degree, marine stratocumulus satisfies these criteria. Spaceborne retrievals of CDNC, LWP, and cloud cover, and possibly other variables (e.g., drizzle from Cloudsat), can be used for model evaluation. CDNC could serve as a proxy for the aerosol variability. In principle, models could be used to calculate the radiances actually measured by satellites.

\section{Ensembles of LES/CRM Model Simulations}

The understanding of aerosol-cloud interactions at a large scale might be advanced by simulating broad ranges of meteorological situations with process models. LESs are highly idealized, limited domain (order of $10 \mathrm{~km}$ ) simulations. When embedding LES within a regional model, mesoscale forcing is provided to the finer LES grid. Two-way nesting allows for communication between grids. In a different approach, meteorological parameters, such as SST 
and large-scale subsidence, may be varied along with aerosol perturbations to explore the relative importance of aerosol and meteorology.

A multiscale modeling framework, which replaces the conventional cloud parameterizations with a CRM in each grid-column of a general circulation model, or global CRMs are ideal tools to apply CRMs to a large variety of meteorological situations for all regions of the globe. These could be used to investigate the effects of indirect aerosols on a large scale (Collins and Satoh; Grabowski and Petch, both this volume).

\section{Improving Large-scale Model Parameterizations}

It has been a long-standing goal to use field programs, process models, and satellite observations effectively to improve GCM parameterizations, which are of critical importance to represent the effects of indirect aerosols accurately. Still, this remains a daunting challenge. A basic strategy to achieve this goal is first to develop confidence in process models (e.g., LES, CRMs). Field campaigns can play a central role in doing this. Next, process models must be used in the parameterization process. Finally, satellite observations, including statistical analysis of covariances, provide a means to evaluate the GCM with its parameterization.

\section{Ice and Mixed-phase Clouds}

Much of the previous discussion centered on the link from aerosols to the CDNC and albedo of warm clouds. Whereas warm clouds (extensive stratus or stratocumulus sheets especially over the oceans) are important for the radiation balance, mixed-phase and ice clouds are more important in terms of the possible effect of aerosols on precipitation. However, there are much larger uncertainties associated with the processes acting in these clouds.

\section{Summary}

Various studies of observational data have established statistical relationships between cloud microphysical properties and aerosol concentrations consistent with an assumed aerosol indirect effect. However, covariance of aerosol concentrations and meteorological cloud-controlling factors leads to a variety of responses beyond the enhancement of CDNC at increased aerosol concentrations. Time history in 3-D data is needed to overcome some of the issues. To interpret correlations found in observational data, process model studies can establish causality, whereas process models applied to larger scales can be used to comprehend aerosol effects beyond instantaneous influences. A better understanding of models at the process scale may help us improve parameterizations in large-scale models. 


\section{Is the Concept of Forcing for Aerosols Useful? If Not, What Is a Viable Definition of Forcing?}

\section{Shortcomings of IPCC's Radiative Forcing Concept}

The forcing summary graph put forth by the IPCC (2007) includes only the direct forcing and Twomey aerosol indirect effect. It does not reflect the full magnitude or uncertainty of cloud-mediated aerosol forcing. However, some effects (e.g., the aerosol "cloud lifetime effect,") for which confidence in understanding is limited do not necessarily have the smallest magnitude, and therefore they cannot be omitted. The inclusion of such uncertain effects emphasizes the imperative need to narrow the gap in our understanding.

Forcing, as used by IPCC, is well-defined for the cloud albedo effect when simulated in a simplified manner, but it cannot capture the microphysical responses to aerosol-related changes in cloud particle number. For well-mixed greenhouse gases, a consistent scaling between forcing and climate change is a reasonable expectation, but this is less so for agents such as aerosols, which are not well-mixed. Aerosol-cloud interactions break this consistency further (Haywood et al., this volume).

\section{The Radiative Flux Perturbation as an Alternative}

Standard radiative forcing is computed as the change in radiation fluxes at the tropopause attibutable to an external perturbation, with tropospheric temperature and humidity profiles held fixed, but allowing for fast (order of months) temperature adjustment in the stratosphere. In contrast, radiative flux perturbation allows for rapidly responding tropospheric meteorological fields to adjust as well. The radiative flux perturbation concept does amount to "fixed-SST forcing" (Shine et al. 2003; Hansen et al. 2005; Forster et al. 2007) or "quasiforcing" (Rotstayn and Penner 2001).

The radiative flux perturbation is likely to yield a more consistent scaling with climate change than forcing. It is also likely to convey more intermodel uncertainty than forcing, thus including some information about the level of scientific uncertainty.

Recent work suggests that one of the consequences of adopting a flux perturbation approach for the assessment of greenhouse gas forcing is that the flux perturbation may include a significant cloud response component (Gregory and Webb 2008; Andrews and Forster 2008). This work further suggests that the cloud-climate feedback, when strictly defined as being the response of clouds to increases in surface temperature, may actually be quite small. Thus, uncertainty in the proportionality between radiative flux perturbation and global mean change in surface temperature would probably be less than for the proportionality between forcing and global mean temperature change. Of course, this inclusion of response to forcing in the radiative flux perturbation does not 
reduce overall uncertainty in, for example, expected warming at the end of the $21^{\text {st }}$ century.

The proposal to adopt the radiative flux perturbation concept for aerosol radiative impacts implies the convolution of direct and indirect effects, rapid cloud responses, and other rapid climate responses in a single measure. This convolution is similar to the mixing of forcing and response in the actual climate system. Observational estimates of flux perturbations should be based on total derivatives of regional and global planetary albedo with respect to measurable extrinsic aerosol properties, such as optical depth. Since the flux perturbation is based upon fixed SSTs, it might be useful to composite oceanic observations with similar spatial patterns of SST but varying measures of aerosol loading. Since the flux perturbation includes the radiative adjustment of the troposphere to anthropogenic aerosol perturbations, it may be necessary to combine observations over the appropriate radiative dynamic timescales.

Aerosol indirect effects other than the Twomey effect are difficult to define. The distinction between first, second, and semi-direct effects is obsolete, and many more cloud-aerosol interaction "effects" may be defined. Thus, the replacement of various ill-defined aerosol indirect effects by just the combined radiative flux perturbation quantification appears to be an advantage of this concept.

\section{Open Issues}

Whether radiative flux perturbations exhibit the same additivity as practiced for traditional radiative forcings remains unknown. The relationships between flux perturbations and traditional forcings for greenhouse gases and aerosols need to be quantified across the ensemble of climate models used for IPCC.

To calculate forcing (as precisely defined), GCM simulations using a double-call to the radiation scheme need to run typically for only one year in order to sample the full seasonal cycle. In contrast, simulations to calculate the flux perturbation must last considerably longer, typically from $5-10$ years. This is because the meteorology usually differs between control and perturbed simulations in calculating the flux perturbation, and thus one has to run the simulations for a number of years to obtain a good signal-to-noise ratio.

One difficulty with the flux perturbation is that usually only SSTs and seaice extents are prescribed. This means that although feedbacks via surface temperature over ocean areas are eliminated, those via changes in land-surface temperature are not; neither are other land-surface responses, such as changes in soil moisture or snow cover. Ideally, land-surface temperatures would also be prescribed, but the complex formulation of land-surface parameterization schemes in current GCMs makes this difficult and may not be possible for all models. Thus, we suggest the flux perturbation in the form of "fixed-SST forcing." 


\section{Summary}

We recommend that the community quantify the effects of aerosols on climate using the concept of radiative flux perturbations, known in the literature as quasi-forcing or fixed-SST forcing. Although extremely useful for well-mixed greenhouse gases, the concept of forcing has limited utility and conceptual rigor when applied to cloud-aerosol interactions. Since the comparison of forcings from various radiative species has been very useful for scientific and policy-oriented applications, we recommend, in addition, that the community compute the effects of all anthropogenic agents using the radiative flux perturbation approach. The traditional IPCC bar chart for forcings could be complemented by a corresponding bar chart for radiative flux perturbations. The application of the flux perturbation concept includes the advantage of a more intuitive observational assessment possibility, a tighter definition of feedbacks as a pure response to surface temperature warming, and a more plausible definition of aerosol effects.

\section{References}

Anderson, T. L., R. J. Charlson, S. E. Schwartz et al. 2003. Climate forcing by aerosols: A hazy picture. Science 300:1103-1104.

Andrews, T., and P. M. Forster. 2008. $\mathrm{CO}_{2}$ forcing induces semi-direct effects with consequences for climate feedback interpretations. Geophys. Res. Lett. 35:L04802.

Bodas-Salcedo, A., M. J. Webb, M. E. Brooks et al. 2008. Evaluation of cloud systems in the Met Office global forecast model using CloudSat data. Geophy. Res. Abst. 10:EGU2008-A-10141.

Bony, S., J.-L. Dufresne, H. Le Treut, J.-J. Morcrette, and C. Senior. 2004. On dynamic and thermodynamic components of cloud changes, Climate Dyn. 22:71-86.

Browning, K. A., et al. 1993. The GEWEX Cloud System Study (GCSS). Bull. Amer. Meteor. Soc. 74:387-399.

Cess, R. D., G. L. Potter, J. P. Blanchet et al. 1990. Intercomparison and interpretation of climate feedback processes in 19 atmospheric general circulation models. J. Geophys. Res. 95(D10):16,601-16,615.

Chepfer, H., S. Bony, M. Chiriaco et al. 2008. Use of CALIPSO lidar observations to evaluate the cloudiness simulated by a climate model. Geophys. Res. Lett. 35:L15804.

Coakley, J. A., P. A. Durkee, K. Nielsen et al. 2000. The appearance and disappearance of ship tracks on large spatial scales. J. Atmos. Sci. 57:2765-2778.

Denman, K. L., G. Brasseur, A. Chidthaisong et al. 2007. Couplings between changes in the climate system and biogeochemistry. In: Climate Change 2007: The Physical Science Basis. Contribution of Working Group I to the Fourth Assessment Report of the Intergovernmental Panel on Climate Change, ed. S. Solomon, D. Qin, M. Manning et al., pp. 499-588. New York: Cambridge Univ. Press.

Dufresne, J.-L., and S. Bony. 2008. An assessment of the primary sources of spread of global warming estimates from coupled ocean-atmosphere models. J. Climate, in press. 
Forster, P., V. Ramaswamy, Artaxo et al. 2007. Radiative Forcing of Climate Change. In: Climate Change 2007: The Physical Science Basis. Contribution of Working Group I to the Fourth Assessment Report of the Intergovernmental Panel on Climate Change, edited by S. Solomon, D. Qin, M. Manning et al., pp. 129-234. New York: Cambridge Univ. Press.

Gregory, J. M., and M. J. Webb. 2008. Tropospheric adjustment induces a cloud component in $\mathrm{CO}_{2}$ forcing. J. Climate. 21(1):58-71.

Hall, A., and X. Qu. 2006. Using the current seasonal cycle to constrain snow albedo feedback in future climate change. Geophys. Res. Lett. 33:L03502.

Hansen, J., M. Sato, R. Ruedy et al. 2005. Efficacy of climate forcings. J. Geophys. Res. 110:D18104.

Illingworth, A. J., R. J. Hogan, E. J. O'Connor et al. 2007. Cloudnet: Continuous evaluation of cloud profiles in seven operational models using ground-based observations. Bull. Am. Meteor. Soc. 88:883-898.

IPCC. 2007. Climate Change 2007: The Physical Science Basis. Contribution of Working Group I to the Fourth Assessment Report of the Intergovernmental Panel on Climate Change, ed. S. Solomon, D. Qin, M. Manning et al. New York: Cambridge Univ. Press.

Leith, C. E. 1975. Climate response and fluctuation dissipation. J. Atmos. Sci. 32:20222026.

Loeb, N. G., B. A. Wielicki, W. Su et al. 2007. Multi-instrument comparison of top of the atmosphere reflected solar radiation, J. Climate 20:575-591.

Neelin, J. D., and N. Zeng. 2000. A quasi-equilibrium tropical circulation modelformulation. J. Atmos. Sci. 57:1741-1766.

Rodwell, M. J., and T. N. Palmer. 2007. Using numerical weather prediction to assess climate models. Q. J. Roy. Meteor. Soc. 133:129-146.

Rotstayn, L. D., and J. E. Penner. 2001. Indirect aerosol forcing, quasi forcing, and climate response. J. Climate 14:2960-2975.

Schwartz, S. E. 2007. Heat capacity, time constant, and sensitivity of Earth's climate system. J. Geophys. Res. 112:D24S05.

Shine, K. P., J. Cook, E. J. Highwood, and M. M. Joshi. 2003. An alternative to radiative forcing for estimating the relative importance of climate change mechanisms. Geophys. Res. Lett. 30:2047.

Soden, B. J., and I. M. Held. 2006. An assessment of climate feedbacks in coupled ocean-atmosphere models. J. Climate 19:3354-3360.

Soden, B. J., R. T. Wetherald, G. L. Stenchikov, and A. Robock. 2002. Global cooling after the eruption of Mount Pinatubo: A test of climate feedback by water vapor. Science 296:727.

Twomey, S. A. 1974. Pollution and the planetary albedo. Atmos. Environ. 8:12511256.

Webb, M., C. Senior, S. Bony, and J.-J. Morcrette. 2001. Combining ERBE and ISCCP data to assess clouds in the Hadley Centre, ECMWF and LMD atmospheric climate models. Climate Dyn. 17:905-922.

Williams, K. D., and G. Tselioudis. 2007. GCM intercomparison of global cloud regimes: Present-day evaluation and climate change response. Climate Dyn. 29:231-250.

Yokohata, T., S. Emori, T. Nozawa et al. 2005. Climate response to volcanic forcing: Validation of climate sensitivity of a coupled atmosphere-ocean general circulation model. Geophys. Res. Lett. 32:L21710. 
